\title{
Article
}

\section{NGC 3628-ucd1: a possible w cen analog embedded in a stellar stream}

Jennings, ZG, Romanowsky, AJ, Brodie, JP, Janz, J, Norris, MA, Forbes, DA, Martinez-Delgado, D, Fagioli, M and Penny, S]

Available at http://clok.uclan.ac.uk/15615/

Jennings, ZG, Romanowsky, AJ, Brodie, JP, Janz, J, Norris, MA ORCID: 00000002-7001-805X, Forbes, DA, Martinez-Delgado, D, Fagioli, M and Penny, SJ (2015) NGC 3628-ucd1: a possible $\omega$ cen analog embedded in a stellar stream. The Astrophysical Journal Letters, 812 . p. 6. ISSN 0004-637X

It is advisable to refer to the publisher's version if you intend to cite from the work. http://dx.doi.org/10.1088/2041-8205/812/1/L10

For more information about UCLan's research in this area go to http://www.uclan.ac.uk/researchgroups/ and search for <name of research Group>.

For information about Research generally at UCLan please go to http://www.uclan.ac.uk/research/

All outputs in CLoK are protected by Intellectual Property Rights law, including Copyright law. Copyright, IPR and Moral Rights for the works on this site are retained by the individual authors and/or other copyright owners. Terms and conditions for use of this material are defined in the policies page. 
DRAFT VERSION SEPTEMBER 17, 2015

Preprint typeset using LTEX style emulateapj v. 5/2/11

\title{
NGC 3628-UCD1: A POSSIBLE $\omega$ CEN ANALOG EMBEDDED IN A STELLAR STREAM
}

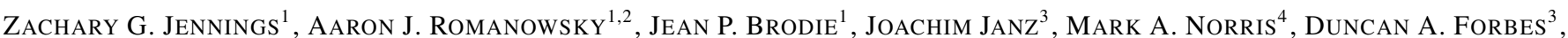

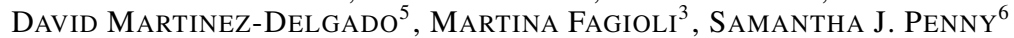 \\ Draft version September 17, 2015
}

\begin{abstract}
Using Subaru/Suprime-Cam wide-field imaging and both Keck/ESI and LBT/MODS spectroscopy, we identify and characterize a compact star cluster, which we term NGC 3628-UCD1, embedded in a stellar stream around the spiral galaxy NGC 3628. The size and luminosity of UCD1 are similar to $\omega$ Cen, the most luminous Milky Way globular cluster, which has long been suspected to be the stripped remnant of an accreted dwarf galaxy. The object has a magnitude of $i=19.3$ mag $\left(\mathrm{L}_{\mathrm{i}}=1.4 \times 10^{6} \mathrm{~L}_{\odot}\right)$. UCD1 is marginally resolved in our ground-based imaging, with a half-light radius of $\sim 10 \mathrm{pc}$. We measure an integrated brightness for the stellar stream of $i=13.1 \mathrm{mag}$, with $(g-i)=1.0$. This would correspond to an accreted dwarf galaxy with an approximate luminosity of $\mathrm{L}_{i} \sim 4.1 \times 10^{8} \mathrm{~L} \odot$. Spectral analysis reveals that UCD1 has an age of $6.6 \mathrm{Gyr}$, $[\mathrm{Z} / \mathrm{H}]=-0.75$, and $[\alpha / \mathrm{Fe}]=-0.10$. We propose that UCD1 is an example of an $\omega$ Cen-like star cluster possibly forming from the nucleus of an infalling dwarf galaxy, demonstrating that at least some of the massive star cluster population may be created through tidal stripping.

Subject headings: galaxies: star clusters: general, galaxies: interactions, galaxies: individual: NGC 3628
\end{abstract}

\section{INTRODUCTION}

Since the discovery of ultra-compact dwarfs (UCDs) a decade and a half ago (Hilker et al. 1999, Drinkwater et al. 2000), there has been considerable discussion in the literature regarding their origin. The conversation can be distilled down to a simple question: are UCDs the largest star clusters, or the smallest galaxies?

The earliest UCDs discovered have $\mathrm{R}_{\mathrm{h}} \sim 20 \mathrm{pc}$ and luminosities $>10^{7} \mathrm{~L}_{\odot}$. These objects represent a middle-ground between globular clusters (GCs), which have $\mathrm{R}_{\mathrm{h}}$ of $\sim 3 \mathrm{pc}$ and luminosities of $\sim 10^{6} \mathrm{~L}_{\odot}$, and dwarf galaxies, with $\mathrm{R}_{\mathrm{h}}>100$ pc. Expanded observational studies have found that UCDs occupy a sequence with similar luminosity to GCs, but larger $\mathrm{R}_{\mathrm{h}}$ (Brodie et al. 2011; Misgeld \& Hilker 2011; Norris et al. 2014). We adopt the definition of UCDs from Brodie et al. (2011): UCDs are objects with $\mathrm{R}_{\mathrm{h}}$ ranging from $\sim 10$ to 100 pc, and luminosities $\mathrm{M}_{\mathrm{i}}<-8.5 \mathrm{mag}$ ( or $\mathrm{L}_{\mathrm{i}} \gtrsim 10^{5} \mathrm{~L}_{\odot}$ ).

The simplistic galaxy vs. cluster distinction breaks down further. UCDs could include objects resulting from mergers of globular clusters (e.g. Fellhauer \& Kroupa 2002; KisslerPatig et al. 2006), or objects formed primordially in intense star formation episodes (Murray 2009). Characterizing the UCD population would have implications for cluster formation physics. In the galaxy scenario, these objects could form primordially in association with distinct dark-matter halos, or they could be the remnant nuclei of larger galaxies which have undergone tidal stripping during accretion onto larger galaxy halos (e.g. Bekki et al.|2001; Pfeffer \& Baumgardt 2013). Un-

\footnotetext{
${ }^{1}$ University of California Observatories, Santa Cruz, CA 95064, USA; zgjennin@ucsc.edu

${ }_{2}^{2}$ Department of Physics and Astronomy, San José State University, One Washington Square, San Jose, CA, 95192, USA

${ }^{3}$ Centre for Astrophysics \& Supercomputing, Swinburne University, Hawthorn VIC 3122

${ }^{4}$ Max Planck Institut für Astronomie, Königstuhl 17, D-69117 Heidelberg, Germany

${ }^{5}$ Astronomisches Rechen-Institut, Zentrum für Astronomie der Universität Heidelberg, Mönchhofstr. 12-14, 69120 Heidelberg, Germany

${ }^{6}$ Institute of Cosmology and Gravitation, University of Portsmouth, Dennis Sciama Building, Burnaby Road, Portsmouth PO1 3FX, UK
}

derstanding the origins of the UCD population has important implications for understanding sub-halos in a $\Lambda$ CDM context.

The most massive Milky Way (MW) GC, $\omega$ Cen, is an outlier among the MW population and may be an example of a sripped dwarf-galaxy nucleus. The cluster has a large velocity dispersion (e.g. Anderson \& van der Marel 2010), rapid rotation leading to flattening (e.g. Merritt et al. 1997), unusual abundance patterns revealing multiple populations (e.g. King et al. 2012), and odd orbital properties (e.g. Dinescu et al. 1999). However, definite confirmation of this formation scenario has remained elusive.

In this work, we identify and describe a star cluster, which we call NGC 3628-UCD1 (hereafter UCD1), embedded in a stellar stream around the nearby spiral galaxy NGC 3628. NGC 3628 is an Sb galaxy with $\mathrm{M}_{\mathrm{V}}=-21.37$. It is located in the Leo Triplet, a loose group with two other large companions, NGC 3623 and NGC 3627. An obvious stellar stream extends $\sim 140 \mathrm{kpc}$ away from the galaxy, shown in Fig. 1. UCD1 is located within the plume nearest to NGC 3628. As shown in $\S 2$, UCD1's size and luminosity are very similar to those of $\omega$ Cen.

The stream itself has been studied extensively since its first characterization (Zwicky 1956, Kormendy \& Bahcall 1974). The stream contains significant neutral hydrogen (Rots 1978; Haynes et al. 1979). Chromey et al. (1998) identified two blue clumps along the stream and estimated young ages for both.

There are several known examples in the literature of UCDs connected with tidal stripping events. Norris \& Kannappan (2011) identified a young UCD around NGC 4546 and argued, based on the properties of the galaxy, that it is a result of stripping. Foster et al. (2014) identified an object likely to be the nucleus of the dwarf galaxy forming the "umbrella stream" around NGC 4651. Mihos et al. (2015) identify a nucleus of an ultra diffuse galaxy in Virgo, which they argue is in the process of tidal threshing.

Using Subaru/Suprime-Cam imaging, we measure photometry and size of both UCD1 and the full stellar stream. We propose that UCD1 is an example of a UCD in formation through tidal stripping. By measuring the UCD and infer- 

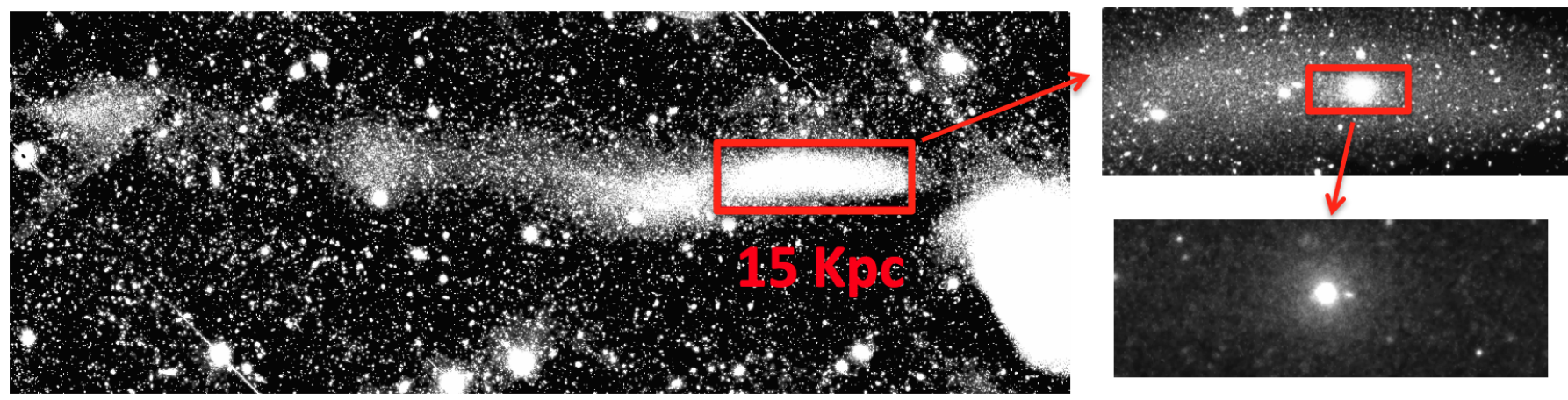

Figure 1. Smoothed image of stellar stream next to NGC 3628 from our $i$-band Subaru/SuprimeCam imaging. The left-most edge of NGC 3628 is visible at the far right of the image. We highlight the location of UCD1 in zoom-in panels. North is up and east is left. The limiting surface brightness in the large image is roughly $\mu_{i} \sim 28.5 \mathrm{mag} \operatorname{arcsecond}^{-2}$. The stretch is modified in each image. Angular sizes are approximately $26 \times 10$ arcmin, $4.5 \times 1.5$ arcmin, and $0.9 \times 0.4$ arcmin from largest to smallest scale.

ring the properties of the potential progenitor galaxy, we are able to draw an evolutionary line in size/luminosity parameter space between the original parent galaxy and the stripped cluster. In $\S 2$, we explain our imaging analysis and results, and in $\S 3$, we do the same for our spectroscopy. We discuss our results in $\$ 4$.

NGC 3627 has a Cepheid distance measurement of 10.6 Mpc (Kanbur et al. 2003). We adopt this value for NGC 3628 and include an approximate distance uncertainty of $\pm 1 \mathrm{Mpc}$ on distance-dependent properties, given the potential offset of NGC 3628 from NGC 3627.

\section{IMAGING}

\subsection{Data Reduction}

We imaged NGC 3628 in $r$-band for 425s on 2009 April 20th with Subaru/Suprime-Cam. UCD1, a marginallyresolved source in the center of plume just east of NGC 3628 , was first noticed in this pointing. We subsequently acquired imaging centered on UCD1 in the $i$-band on 2014 March 3 (425s exposure time) and in the $g$-band on 2014 December 19 (1225s exposure time). Seeing was $\sim 0.80^{\prime \prime}, \sim 0.70^{\prime \prime}$, and $\sim 0.75^{\prime \prime}$ in $g, r, i$ respectively. We employed a modified version of the SDFRED-2 pipeline ${ }^{7}$ to reduce our SuprimeCam data. AB Zeropoints were calculated by comparing photometry for bright, unsaturated stars in both the SDSS cata$\log$ and the Suprime-Cam imaging. We used the Schlafly \& Finkbeiner (2011) values from NED to correct for Galactic extinction.

\subsection{Photometry and Size of UCDI}

We performed aperture photometry of UCD1 using an aperture roughly twice the size of the FWHM for each image, chosen to maximize the $\mathrm{S} / \mathrm{N}$. Aperture corrections were measured using several bright, unsaturated stars in the field. Uncertainties in aperture corrections were $0.04 \mathrm{mag}$ in $g$ and 0.03 mag in $r$ and $i$. Choice of sky subtraction annulus introduced systematic uncertainties of 0.03 in all filters, with no effect on color. Our photometry is listed in Table 1. At our assumed distance, the luminosity of UCD1 is $\mathrm{L}_{\mathrm{i}}=(1.4 \pm 0.2) \times$ $10^{6} \mathrm{~L}_{\odot}$ with the uncertainty dominated by the distance. The color measured for UCD1 is dependent on the aperture selected. When an aperture equal to the FWHM is used, the color is $g-i=0.86$, which is comparable to $g-i=0.91$ inferred from the stellar populations in the ESI spectrum (see $\S 3)$. Note that the seeing FWHM in our imaging is roughly

\begin{tabular}{|c|c|c|}
\hline Parameter & $\begin{array}{c}\text { JCD1 Properties } \\
\text { Value }\end{array}$ & Uncertainty \\
\hline R.A. (J2000, from SDSS) & 170.25493 & - \\
\hline Dec $(\mathrm{J} 2000$, from SDSS $)$ & 13.60813 & - \\
\hline \multicolumn{3}{|c|}{ Suprime-Cam Photometry } \\
\hline$g$ & $19.98 \mathrm{mag}$ & $0.05 \mathrm{mag}$ \\
\hline$r$ & $19.57 \mathrm{mag}$ & $0.04 \mathrm{mag}$ \\
\hline$i$ & $19.29 \mathrm{mag}$ & $0.04 \mathrm{mag}$ \\
\hline$(g-r)$ & $0.41 \mathrm{mag}$ & $0.05 \mathrm{mag}$ \\
\hline$(g-i)$ & $0.69 \mathrm{mag}$ & $0.05 \mathrm{mag}$ \\
\hline $\mathrm{R}_{\mathrm{h}}, r$-band & $10 \mathrm{pc}$ & $3 \mathrm{pc}$ \\
\hline Ellipticity & 0.9 & - \\
\hline Luminosity & $\mathrm{L}_{\mathrm{i}}=1.4 \times 10^{6} \mathrm{~L} \odot$ & $\pm 0.2 \times 10^{6} \mathrm{~L}_{\odot}$ \\
\hline \multicolumn{3}{|c|}{ ESI Spectroscopy } \\
\hline Vel. & $815 \mathrm{~km} \mathrm{~s}^{-1}$ & $4 \mathrm{~km} \mathrm{~s}^{-1}$ \\
\hline Vel. Dispersion & $\lesssim 23 \mathrm{~km} \mathrm{~s}^{-1}$ & - \\
\hline Age & $6.6 \mathrm{Gyr}$ & $+1.9 \mathrm{Gyr}$ \\
\hline$[\mathrm{Z} / \mathrm{H}]$ & -0.75 & 0.12 \\
\hline$[\alpha / \mathrm{Fe}]$ & -0.10 & 0.08 \\
\hline \multicolumn{3}{|c|}{ MODS Spectroscopy } \\
\hline Age & $6.6 \mathrm{Gyr}$ & $+1.4 \mathrm{Gyr}$ \\
\hline$[\mathrm{Z} / \mathrm{H}]$ & -0.77 & 0.16 \\
\hline$[\alpha / \mathrm{Fe}]$ & -0.08 & 0.15 \\
\hline
\end{tabular}

\begin{tabular}{ccc} 
& $\begin{array}{r}\text { Surface Photometry } \\
\text { Apparent Mag }\end{array}$ & Luminosity \\
\hline$g$ & Full Stream & \\
$i$ & $14.15 \mathrm{mag}$ & $(2.7 \pm 0.5) \times 10^{8} \mathrm{~L} \odot$ \\
$(g-i)$ & $13.14 \mathrm{mag}$ & $(4.1 \pm 0.8) \times 10^{8} \mathrm{~L} \odot$ \\
& $1.01 \mathrm{mag}$ & - \\
$g$ & Plume Containing UCD1 & $\left(9 ._{-1.7}^{+1.8}\right) \times 10^{7} \mathrm{~L} \odot$ \\
$i$ & $15.34 \mathrm{mag}$ & $(1.6 \pm 0.3) \times 10^{8} \mathrm{~L} \odot$ \\
$(g-i)$ & $14.19 \mathrm{mag}$ & -
\end{tabular}

comparable to the width of the ESI slit used. Using the max $\mathrm{S} / \mathrm{N}$ aperture results in bluer colors $(g-i=0.69)$. This effect may be due to contamination by the stream, or could be caused by some gradient intrinsic to UCD1. Robust determination of UCD1's color will require more sophisticated stream/source decomposition. Given the varying quality of our ground-based images, our current dataset is not wellsuited to this task.

UCD1 is marginally resolved in our imaging; we used ishape (Larsen 1999) to measure $\mathrm{R}_{\mathrm{h}}$, exploring Sersic and King profile fits. ishape convolves a model light profile with an empirical PSF and fits it to the source. We measured the

\footnotetext{
${ }^{7}$ http://subarutelescope.org/Observing/Instruments/SCam/sdfred/sdfred2.html.enPSF from bright, unsaturated stars in the FOV. Both Sersic
} 
and King profiles feature a parameter to describe the shape of the profile. When left completely free, the resulting fits featured unphysical values for shape parameters. However, we found that varying these parameters over a reasonable range changed $\mathrm{R}_{\mathrm{h}}$ at roughly the $20 \%$ level.

Across all filters, a model+PSF was always a better fit than the PSF-only model. As the $r$-band imaging features the best seeing, we adopt our $r$-band fits for UCD1's fiducial $\mathrm{R}_{\mathrm{h}}$. In general, fits to the $i$-band data tended to be $\sim 10 \%$ smaller, while fits in $g$-band data tended to be $\sim 10 \%$ larger than $r$ band measured $\mathrm{R}_{\mathrm{h}}$.

We find a size of $7.5 \mathrm{pc}$ for a $c=15$ a King profile and 12.5 pc for an $n=4$ Sersic profile The differences in residuals are not large between the two assumed models. We ultimately choose to adopt the average of the two values and note a \pm 2 pc systematic uncertainty on this measurement depending on the choice of profile. We also note the $10 \%$ uncertainty from our assumed distance, as well as $\sim 20 \%$ scatter for choices of concentration parameter. Our final $r$-band $\mathrm{R}_{\mathrm{h}}$ estimate for UCD1 is thus $10 \pm 3 \mathrm{pc}$.

\subsection{Surface Photometry of NGC 3628 Stream}

One possible explanation for the stellar stream is the accretion of a dwarf galaxy. If this scenario is true, then the total luminosity of the NGC 3628 stream offers a useful constraint on the luminosity of this accreted dwarf.

The Suprime-Cam reduction pipeline subtracts a constant sky brightness off the image. We use SExtractor to model any remaining varying background, adopting a mesh size of 512 pixels. This size was chosen to be significantly larger than the stream. We inspected the background maps to verify the stream was not being included. Bright objects were masked in the imaging. We then use adaptsmooth (Zibetti 2009; Zibetti et al.2009) to perform adaptive smoothing to a uniform signal to noise ratio $(\mathrm{S} / \mathrm{N})$ on the background-subtracted images. We select a $\mathrm{S} / \mathrm{N}$ threshold of five (limiting surface brightness of $\mu_{i} \sim 28.5$ mag $\operatorname{arcsecond}^{-2}$ ).

We performed aperture photometry of the stream in this smoothed image using a custom aperture (the same for all filters) and the polyphot task in IRAF, with pixels below the $\mathrm{S} / \mathrm{N}$ threshold excluded from the measurement. The resulting measurements are listed in Table 2.

We measure a total apparent $i$-band magnitude of 13.14 . Statistical uncertainties are small (a few thousandths of a magnitude), but doubling or halving the required signal-tonoise modifies the final answer by around $0.1 \mathrm{mag}$. We adopt $\pm 0.1 \mathrm{mag}$ as a rough estimate of the systematic uncertainty in the measurement, which is smaller than the distance uncertainty $( \pm 0.2 \mathrm{mag})$. The total luminosity of the stream is $\mathrm{L}_{i} \simeq(4.1 \pm 0.8) \times 10^{8} \mathrm{~L}_{\odot}$. We emphasize that this estimate could miss additional starlight below our detection threshold or behind NGC 3628, or could include contamination from faint contaminant point sources, and therefore should be regarded with caution when interpreted directly as the accreted galaxy luminosity. We measure $g-i=1.01$ for the color of the full stream.

The plume which contains UCD1 has $i=14.19$ and $g=$ 15.34 , giving $(g-i)=1.15$. The approximate area used for the aperture photometry is marked in Fig. 1. Using the galaxy stellar population models of Into \& Portinari (2013), the corresponding $i$-band stellar mass-to-light ratio $\left(\mathrm{M} / \mathrm{L}_{*}\right)$ is $2.5-$ 2.9. This color may be more representative of the stellar population of the progenitor dwarf galaxy, as the full-stream mea-

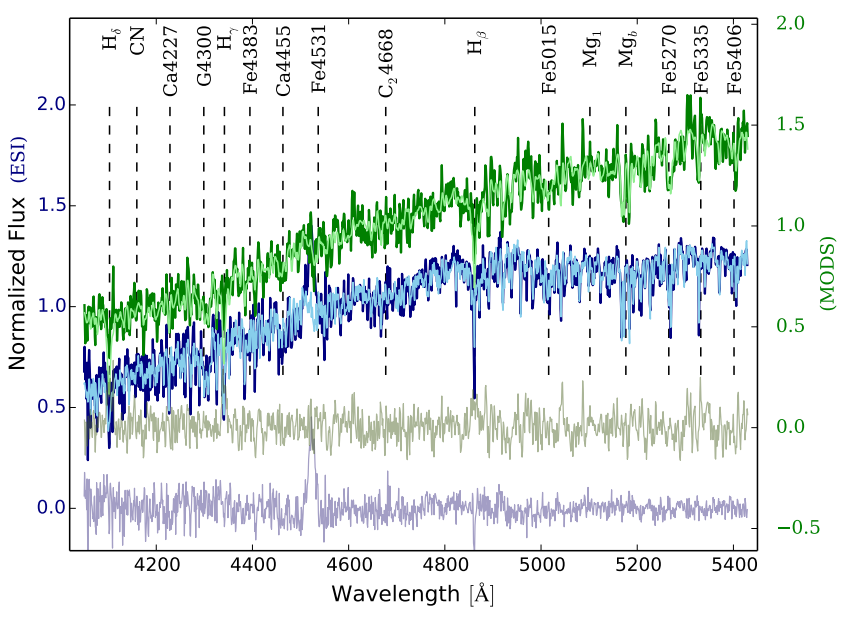

Figure 2. Keck/ESI and LBT/MODS spectra of UCD1. ESI data are shown in dark blue, while MODS data are shown in dark green. Light blue and light green represent model fits to both spectra. Residuals are shown at the bottom. The MODS scale is offset from the ESI scale.

surement can be more sensitive to contaminants and choice of aperture. Both the plume and full stream color and luminosity measurements fall on the red side of galactic scaling relations from Janz \& Lisker (2009), but they still are broadly within the scatter.

\section{SPECTROSCOPY OF UCD1}

A 3600s KECK/ESI spectrum of NGC3628 UCD1 was acquired on 2014 March 20th with the $0.75^{\prime \prime}$ longslit, with a S/N of $\sim 23 \AA^{-1}$. We subsequently acquired a 2400s LBT/MODS spectrum on 2015 June 6th with the $0.80^{\prime \prime}$ longslit, with a S/N of $\sim 15 \AA^{-1}$. We follow the same procedure as Janz et al. (2015) to measure stellar populations. Briefly, we measure all Lick indices. These are then compared to the single stellar population models of Thomas et al. (2011), which give the best age, metallicity and alpha element abundance via a $\chi^{2}$ minimization process. Poorly fitting lines are excluded from the analysis in an iterative way. The final fit and residuals to both the observed spectra are shown in Fig. 2 .

For the ESI spectrum, we find an old age of $6.6_{-1.5}^{+1.9} \mathrm{Gyr}$ and a metal-poor population of $[\mathrm{Z} / \mathrm{H}]=-0.75 \pm 0.12$, and alphaelement abundance of $[\alpha / \mathrm{Fe}]=-0.10 \pm 0.08$. The MODS spectrum gives consistent results, with an age of , $6.6_{-1.2}^{+1.4} \mathrm{Gyr}$, $[\mathrm{Z} / \mathrm{H}]=-0.77 \pm 0.16$ and $[\alpha / \mathrm{Fe}]=-0.08 \pm 0.15$. Using the relation $[\mathrm{Fe} / \mathrm{H}]=[\mathrm{Z} / \mathrm{H}]-0.94 \times[\alpha / \mathrm{Fe}]($ Thomas et al. 2003), we find $[\mathrm{Fe} / \mathrm{H}]=-0.84$ for the ESI data.

As the ESI spectrum has higher resolution, we use it to measure kinematics (MODS is limited to $\sigma \sim 55 \mathrm{~km} \mathrm{~s}^{-1}$ ). We measure a heliocentric velocity of $815 \pm 4 \mathrm{~km} \mathrm{~s}^{-1}$. While we find a best-fit velocity dispersion of $10.5 \mathrm{~km} \mathrm{~s}^{-1}$, this measurement is significantly below the resolution of the ESI spectrograph $\left(\sigma=23 \mathrm{~km} \mathrm{~s}^{-1}\right)$. Velocity dispersions so low include unquantified systematics, and it is unclear if meaningful constraint can be obtained that low (see also Geha et al. 2002; Janz et al. 2015). As a result, we adopt $\sigma=23 \mathrm{~km} \mathrm{~s}^{-1}$ as the upper limit for the velocity dispersion.

We may make an estimate of the dynamical mass of UCD1 using the expression $M_{\text {dyn }}=C G^{-1} \sigma^{2} R$, where $R$ is taken to be the half-light radius and $C$ is the viral coefficient. We follow Forbes et al. (2014) and adopt a value of 6.5, although values between 4 to 7.5 are reasonable. Given this expression and 
using our measured size of $10 \mathrm{pc}$, we estimate $M_{\text {dyn }}$ to be less than $\sim 8 \times 10^{6} \mathrm{M}_{\odot}$.

There is a significant offset of $\sim 75 \mathrm{~km} \mathrm{~s}^{-1}$ between the measured heliocentric velocity of UCD1 and the heliocentric velocity measured from the $\mathrm{HI}$ gas in the stream. NikielWroczyński et al. (2014) found a gas velocity of $\sim 890 \mathrm{~km}$ $\mathrm{s}^{-1}$ in the vicinity of UCD1. While this could indicate that UCD1 is not associated with the stream, we find this unlikely. UCD1 appears directly at the center of the brightest plume in the stream, and displays a blotchy morphology similar to that in the stream itself. UCD1 is the brightest point in the stream, with the surface brightness of the stream falling off slowly in all directions away from UCD1. It is unclear that we would expect the velocity of the HI gas to follow that of the stream; the gas obeys different physics than the stars in the stream (e.g. ram pressure stripping), and so it would not be surprising to see a velocity offset between the two. UCD1 is blue-shifted by $\sim 30 \mathrm{~km} \mathrm{~s}^{-1}$ compared to NGC 3628 , while the gas is red-shifted by $\sim 45 \mathrm{~km} \mathrm{~s}^{-1}$.

\section{DISCUSSION AND SUMMARY}

\subsection{The Origin of the Tidal Stream}

In the literature, this stellar stream has often been considered the result of an interaction between NGC 3628 and another galaxy in the group, typically NGC 3627. However, the properties of UCD1 give us cause to consider a potential dwarf galaxy minor-merger scenario as the source of the stream.

Rots (1978) carried out a restricted 3-body simulation of a tidal interaction between NGC 3627 and NGC 3628. The simulation did a reasonable job of reproducing some features but also had some discrepancies with observations, such as a smaller velocity difference between the two large galaxies than was actually observed (a full list of issues is enumerated in Haynes et al. 1979). This early simulation also did not take into account other bodies in the system, an important caveat given that the galaxies share a group environment with NGC 3623 to the south east.

Now that we have identified and characterized UCD1, a successful simulation must be able to explain the presence of $\mathrm{a} \sim 10^{6} \mathrm{M}_{\odot}$ compact star cluster with $\mathrm{a} \sim 6 \mathrm{Gyr}$, metal-poor stellar population. It is not immediately obvious how such an object could result simply from the interaction of NGC 3627 and NGC 3628, as we would expect objects resulting from such an interaction to be younger, metal-rich, and less compact. It is important to note that, in this work, we are not making any direct comment on the tidal interaction. We have not yet conducted dynamical modeling of our own, and so we are not in a firm position to endorse a minor-merger picture for this tidal interaction over the NGC 3628/NGC 3627 interaction model currently in the literature. The study of this system would benefit greatly from more sophisticated dynamical modeling, exploring the possibility of minor mergers and including the dynamical effects of all three group galaxies. For the moment, we consider the nature of the merger an open question.

\subsection{UCD1 compared to other UCDs}

It is informative to consider the properties of UCD1 in the context of other UCDs. In the left panel of Fig. 3, we plot the measured half-light radius in pc against the absolute $\mathrm{V}$ band magnitude for a large collection of distance-confirmed dispersion-supported stellar systems across a wide range of sizes and luminosities (Brodie et al. 2014).

In the right panel of Fig. 3, we plot the age and metallicity of various star clusters including confirmed UCDs, MW GCs, nuclear star clusters (NSCs), and early-type galaxy (ETG) centers. We also hightlight an assortment of metal-rich UCDs with a likely tidal stripping origin (e.g. M60-UCD1, see Sandoval et al. 2015).

$\omega$ Cen represents an interesting analogue to UCD1 in these parameters. Its size and luminosity are very similar to UCD1 and both are low metallicity compared to other clusters with a likely tidal origin. The Harris 1996 (2010 edition) catalog lists values of $\sigma=16.8 \mathrm{~km} \mathrm{~s}^{-1}$ (e.g. McLaughlin \& van der Marel 2005), $M_{V}=-10.26$, and $\mathrm{R}_{\mathrm{h}}=7.5 \mathrm{pc}$ (e.g. van den Bergh et al. 1991), all comparable to UCD1 within the uncertainties (note that we only estimate an upper limit for $\sigma$ for UCD1). $\omega$ Cen is even more metal-poor than UCD1, $[\mathrm{Fe} / \mathrm{H}]=-1.53$ (e.g. Johnson et al. 2009) vs. -0.84 , and, generally speaking, is alpha-enhanced (e.g. Johnson \& Pilachowski 2010). Such differences are not unexpected, since UCD1 is only currently being stripped. Given that $\omega$ Cen was likely accreted several Gyr ago, UCD1 is naturally expected to have a more extended SF history, enhancing its metallicity and potentially erasing any alpha-enhancement. In this context, objects like UCD1 may be reasonable modern-day examples of accretion events which, Gyr ago, would have resulted in $\omega$ Cen-like star clusters. These clusters offer interesting contrasts in size, luminosity, and metallicity compared to more metal-rich, tidally stripped UCDs.

\subsection{Properties of a Potential Dwarf Galaxy Progenitor}

In this section, we consider the implications if UCD1 did indeed result from a minor merger. Using the stellar stream luminosity to estimate the stellar mass in the stream is straightforward. As discussed in $\$ 2.3$, for a stream of this color, a $M / L_{*} \sim 2.7$ is reasonable. Given the measured luminosity of $(4.1 \pm 0.8) \times 10^{8} \mathrm{~L}_{\odot}$, we estimate a stellar mass of $\sim 1.1 \times 10^{9} \mathrm{M}_{\odot}$. We take this value as a rough estimate of the stellar mass of a potential accreted dwarf galaxy, also noting neglected systematics from the mass-to-light estimate. This mass is comparable to the spheroid mass estimated in Norris \& Kannappan (2011) for the progenitor of the confirmed stripped nucleus NGC 4546 UCD1 $\left(3.4_{-15}^{+1.2} \times 10^{9} \mathrm{M}_{\odot}\right)$. Following the specific frequencies of Peng et al. (2008), such an accretion event would have contributed $\sim 9$ GCs to the halo of NGC 3628, which could possibly be found as a discrete GC population in phase space.

To estimate a width for the stream, we use a region to the east of UCD1 $\sim 100$ arcseconds wide and sum up the light horizontally along the direction of the stream,. We identify the peak of the light distribution as the stream center, and mark the outer end of the stream as the point where the light profile approximately flattens. Finally, we define the distance from the center that contains half the total integrated light of the region as the half-light width of the stream. We find a value of $\sim 60^{\prime \prime}$, or $\sim 3000 \mathrm{pc}$. We then adopt this half-light width as a proxy for the $R_{h}$ of the accreted parent galaxy. In Fig. 3, we plot the estimated location of this dwarf galaxy in the same parameter space. We emphasize that the connection between stream-width and progenitor size is unclear, and will at the very least depend on viewing angles and orbital phase.

If a minor-merger picture is correct, it is natural to compare UCD1 to nuclear star clusters (NSCs). NSCs typically have 

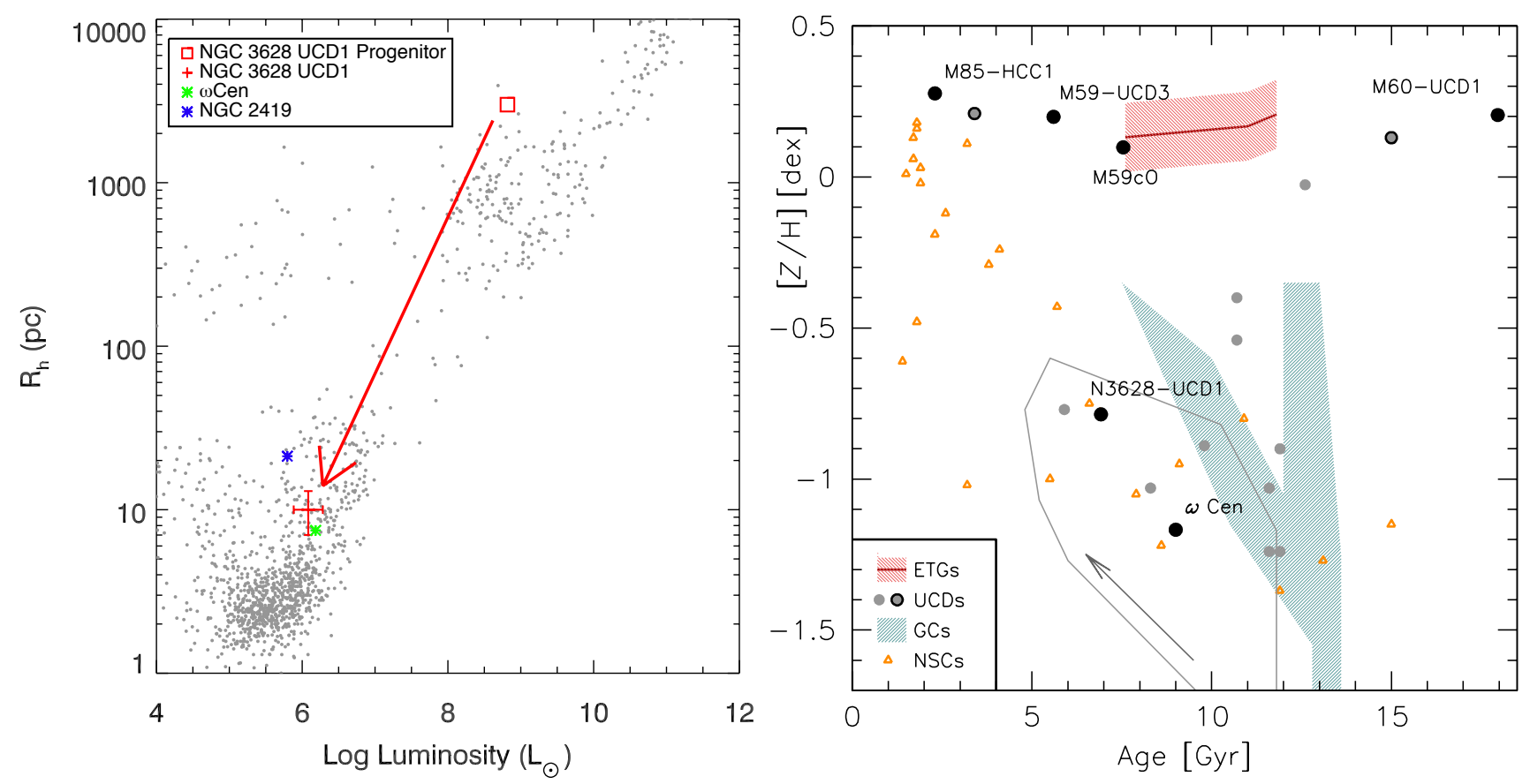

Figure 3. Left panel: Plot of $\mathrm{R}_{\mathrm{h}}$ (pc) vs. $V$-band luminosity $\left(\mathrm{L}_{\odot}\right)$ for a collection of distance-confirmed dispersion supported stellar systems. Data are from a compilation begun in Brodie et al. (2011), updated in Brodie et al. (2014). The location of UCD1 is marked. We also mark the location of the MW GCs $\omega$ Cen and NGC 2419 for comparison. We mark the location where the UCDI progenitor galaxy may have originated based on the stellar stream (note that the box size of this point is arbitrary and not indicative of uncertainty). Right panel: plot of age (Gyr) vs. [Z/H], modified from Sandoval et al. (2015). The grey outline corresponds the approximate ranges in both quantities among stars within $\omega$ Cen, and the arrow indicates the expected direction of evolution (Villanova et al. 2014). UCD1 and $\omega$ Cen are compared to centers of ETGs (red shaded region), MW GCs (green shaded region), confirmed UCDs (grey points), and NSCs (orange triangles). See Pritzl et al. (2005); Brodie et al. (2011); Dotter et al. (2011); Conroy et al. (2014). Clusters with likely tidal-stripping origins are also plotted (see Sandoval et al. 2015

effective radii of a few to tens of parsecs, and luminosities from a few times $10^{5} \mathrm{~L}_{\odot}$ to $\sim 10^{8} \mathrm{~L}_{\odot}$ (Georgiev \& Böker 2014). Early-type galaxies in particular may have more compact NSCs; Côté et al. (2006) found a median $\mathrm{R}_{\mathrm{h}}$ of $4.2 \mathrm{pc}$ for prominent NSCs in early-type galaxies. The precise effects of the stripping process on NSC $\mathrm{R}_{\mathrm{h}}$ are complicated and depend on a wide range of orbital parameters and initial conditions, but for most scenarios, we wouldn't expect drastic changes in cluster $R_{h}$ (Pfeffer \& Baumgardt 2013). Bianchini et al. (2015) found that, as galaxies are stripped, central clusters expand to reach the sizes they would have in isolation, i.e. similar to GC/UCD-sized objects. A late-type nucleated galaxy could be a possible source, though presumably the bulge would need to be red enough to explain the colors seen in UCD1's plume.

For UCD1, if the properties remain roughly comparable, then the stripping process has, at the order of magnitude level, resulted in a decrease of roughly 100 in size and 1000 in luminosity. Similar factors of stripping have been seen in simulations of dwarf-elliptical galaxies (Pfeffer \& Baumgardt 2013), although the precise amounts of mass loss depend on the orbital parameters. In any case, we find the properties of UCD1 consistent with the cluster being the final result of a minor merger, offering support that some portion of the large star cluster population may be created through the tidal stripping of dwarf galaxies.

We thank the anonymous referee for comments which greatly improved the paper. We thank J. Strader, J. Choi, H. Baumgardt, and J. Pfeffer for useful discussions in the course of this work. ZGJ is supported in part by an NSF Graduate Research Fellowship. This work was supported by
NSF grants AST-1109878, AST-1211995, AST-1515084, and AST-1518294. D.A.F. and J.J. thank the ARC for financial support via DP130100388. The LBT is an international collaboration among institutions in the United States, Italy and Germany. LBT Corporation partners are: The University of Arizona on behalf of the Arizona university system; Istituto Nazionale di Astrofisica, Italy; LBT Beteiligungsgesellschaft, Germany, representing the Max-Planck Society, the Astrophysical Institute Potsdam, and Heidelberg University; The Ohio State University, and The Research Corporation, on behalf of The University of Notre Dame, University of Minnesota and University of Virginia. Based in part on data collected at Subaru Telescope, which is operated by the National Astronomical Observatory of Japan. Some of the data presented herein were obtained at the W.M. Keck Observatory, which is operated as a scientific partnership among the California Institute of Technology, the University of California and the National Aeronautics and Space Administration. The Observatory was made possible by the generous financial support of the W.M. Keck Foundation. The authors wish to recognize and acknowledge the very significant cultural role and reverence that the summit of Mauna Kea has always had within the indigenous Hawaiian community. We are most fortunate to have the opportunity to conduct observations from this mountain.

\section{REFERENCES}

Anderson, J., \& van der Marel, R. P. 2010, ApJ, 710, 1032

Bekki, K., Couch, W. J., \& Drinkwater, M. J. 2001, ApJ, 552, L105

Bianchini, P., Renaud, F., Gieles, M., \& Varri, A. L. 2015, MNRAS, 447, L40

Brodie, J. P., Romanowsky, A. J., Strader, J., \& Forbes, D. A. 2011, AJ, 142, 199 
Brodie, J. P., Romanowsky, A. J., Strader, J., et al. 2014, ApJ, 796, 52 Chilingarian, I. V., \& Mamon, G. A. 2008, MNRAS, 385, L83

Chromey, F. R., Elmegreen, D. M., Mandell, A., \& McDermott, J. 1998, AJ, 115,2331

Conroy, C., Graves, G. J., \& van Dokkum, P. G. 2014, ApJ, 780, 33

Côté, P., Piatek, S., Ferrarese, L., et al. 2006, ApJS, 165, 57

Dinescu, D. I., Girard, T. M., \& van Altena, W. F. 1999, AJ, 117, 1792

Dotter, A., Sarajedini, A., \& Anderson, J. 2011, ApJ, 738, 74

Drinkwater, M. J., Jones, J. B., Gregg, M. D., \& Phillipps, S. 2000, PASA, 17, 227

Fellhauer, M., \& Kroupa, P. 2002, MNRAS, 330, 642

Forbes, D. A., Norris, M. A., Strader, J., et al. 2014, MNRAS, 444, 2993

Foster, C., Lux, H., Romanowsky, A. J., et al. 2014, MNRAS, 442, 3544

Geha, M., Guhathakurta, P., \& van der Marel, R. P. 2002, AJ, 124, 3073

Georgiev, I. Y., \& Böker, T. 2014, MNRAS, 441, 3570

Harris, W. E. 1996, AJ, 112, 1487

Haynes, M. P., Giovanelli, R., \& Roberts, M. S. 1979, ApJ, 229, 83

Hilker, M., Infante, L., Vieira, G., Kissler-Patig, M., \& Richtler, T. 1999, A\&AS, 134, 75

Into, T., \& Portinari, L. 2013, MNRAS, 430, 2715

Janz, J., Forbes, D. A., Norris, M. A., et al. 2015, MNRAS, 449, 1716

Janz, J., \& Lisker, T. 2009, ApJ, 696, L102

Johnson, C. I., \& Pilachowski, C. A. 2010, ApJ, 722, 1373

Johnson, C. I., Pilachowski, C. A., Michael Rich, R., \& Fulbright, J. P. 2009 , ApJ, 698, 2048

Kanbur, S. M., Ngeow, C., Nikolaev, S., Tanvir, N. R., \& Hendry, M. A. 2003, A\&A, 411, 361

King, I. R., Bedin, L. R., Cassisi, S., et al. 2012, AJ, 144, 5

Kissler-Patig, M., Jordán, A., \& Bastian, N. 2006, A\&A, 448, 1031
Kormendy, J., \& Bahcall, J. N. 1974, AJ, 79, 671

Larsen, S. S. 1999, A\&AS, 139, 393

McLaughlin, D. E., \& van der Marel, R. P. 2005, ApJS, 161, 304

Merritt, D., Meylan, G., \& Mayor, M. 1997, AJ, 114, 1074

Mihos, J. C., Durrell, P. R., Ferrarese, L., et al. 2015, ApJ, 809, L21

Misgeld, I., \& Hilker, M. 2011, MNRAS, 414, 3699

Murray, N. 2009, ApJ, 691, 946

Nikiel-Wroczyński, B., Soida, M., Bomans, D. J., \& Urbanik, M. 2014, ApJ, 786, 144

Norris, M. A., \& Kannappan, S. J. 2011, MNRAS, 414, 739

Norris, M. A., Kannappan, S. J., Forbes, D. A., et al. 2014, MNRAS, 443, 1151

Peng, E. W., Jordán, A., Côté, P., et al. 2008, ApJ, 681, 197

Pfeffer, J., \& Baumgardt, H. 2013, MNRAS, 433, 1997

Pritzl, B. J., Venn, K. A., \& Irwin, M. 2005, AJ, 130, 2140

Rots, A. H. 1978, AJ, 83, 219

Sandoval, M. A., Vo, R. P., Romanowsky, A. J., et al. 2015, ApJ, 808, L32

Schlafly, E. F., \& Finkbeiner, D. P. 2011, ApJ, 737, 103

Strader, J., Seth, A. C., Forbes, D. A., et al. 2013, ApJ, 775, L6

Thomas, D., Maraston, C., \& Bender, R. 2003, MNRAS, 339, 897

Thomas, D., Maraston, C., \& Johansson, J. 2011, MNRAS, 412, 2183

van den Bergh, S., Morbey, C., \& Pazder, J. 1991, ApJ, 375, 594

Villanova, S., Geisler, D., Gratton, R. G., \& Cassisi, S. 2014, ApJ, 791, 107 Zibetti, S. 2009, ArXiv e-prints, arXiv:0911.4956

Zibetti, S., Charlot, S., \& Rix, H.-W. 2009, MNRAS, 400, 1181

Zwicky, F. 1956, Ergebnisse der exakten Naturwissenschaften, 29, 344 